\title{
Article \\ Characterization, Classification, and Authentication of Polygonatum sibiricum Samples by Volatile Profiles and Flavor Properties
}

\author{
Xile Cheng ${ }^{1}$, Hongyuan Ji ${ }^{1}$, Xiang Cheng ${ }^{2}$, Dongmei Wang ${ }^{3, *}$, Tianshi Li ${ }^{4}$, Kun Ren ${ }^{1}$, Shouhe Qu ${ }^{1}$, Yingni Pan ${ }^{1}$ \\ and Xiaoqiu Liu ${ }^{1}$ * \\ 1 School of Chinese Materia Medica, Shenyang Pharmaceutical University, Shenyang 110016, China; \\ believele@126.com (X.C.); jhy129413@163.com (H.J.); renkun_spu@163.com (K.R.); shouhequ@126.com (S.Q.); \\ panyingni@163.com (Y.P.) \\ 2 School of Pharmacy, Bozhou Vocational and Technical College, Bozhou 236000, China; \\ xiangcheng_bzzy@126.com \\ 3 School of Pharmacy, Shenyang Pharmaceutical University, Shenyang 110016, China \\ 4 Bozhou Yonggang Pieces Factory Co., Ltd., Bozhou 236000, China; lixlixiang@163.com \\ * Correspondence: wangdongmei@syphu.edu.cn (D.W.); liuxiaoqiu@syphu.edu.cn (X.L.); \\ Tel./Fax: +86-24-4352-0719 (D.W.); +86-24-4352-0708 (X.L.)
}

check for updates

Citation: Cheng, X.; Ji, H.; Cheng, X.; Wang, D.; Li, T.; Ren, K.; Qu, S.; Pan, Y.; Liu, X. Characterization, Classification, and Authentication of Polygonatum sibiricum Samples by Volatile Profiles and Flavor Properties. Molecules 2022, 27, 25. https://doi.org/10.3390/ molecules 27010025

Academic Editor: Eugenio Aprea

Received: 13 November 2021

Accepted: 18 December 2021

Published: 21 December 2021

Publisher's Note: MDPI stays neutral with regard to jurisdictional claims in published maps and institutional affiliations.

Copyright: (C) 2021 by the authors. Licensee MDPI, Basel, Switzerland. This article is an open access article distributed under the terms and conditions of the Creative Commons Attribution (CC BY) license (https:// creativecommons.org/licenses/by/ $4.0 /)$.

\begin{abstract}
The importance of monitoring key aroma compounds as food characteristics to solve sample classification and authentication is increasing. The rhizome of Polygonatum sibiricum (PR, Huangjing in Chinese) has great potential to serve as an ingredient of functional foods owing to its tonic effect and flavor properties. In this study, we aimed to characterize and classify PR samples obtained from different processing levels through their volatile profiles and flavor properties by using electronic nose, electronic tongue, and headspace gas chromatography-mass spectrometry. Nine flavor indicators (four odor indicators and five taste indicators) had a strong influence on the classification ability, and a total of 54 volatile compounds were identified in all samples. The traditional Chinese processing method significantly decreased the contents of aldehydes and alkanes, while more ketones, nitrogen heterocycles, alcohols, terpenoids, sulfides, and furans/pyrans were generated in the processing cycle. The results confirmed the potential applicability of volatile profiles and flavor properties for classification of PR samples, and this study provided new insights for determining the processing level in food and pharmaceutical industries based on samples with specific flavor characteristics.
\end{abstract}

Keywords: Polygonatum sibiricum; electronic nose; electronic tongue; gas chromatography-mass spectroscopy; chemometric analysis

\section{Introduction}

In recent years, there has been a growing interest in the medicinal and edible plants as functional foods and health-promoting dietary supplements with remarkable market value. Polygonatum sibiricum Red., a type of traditional medicinal herb and edible plant, is mainly distributed in the northern parts of China (such as Liaoning, Hebei, and Inner Mongolia Provinces), North Korea, and Mongolia [1]. Historically, the rhizome of Polygonatum sibiricum (PR, huangjing in Chinese) was documented as "got the essence of soil, be the first in tonics" in the Compendium of Materia Medica (Ming Dynasty, 1590 A.D.) Huangjing is one of the most popular traditional medicinal herbs with a wide range of beneficial effects such as replenishing Qi, nourishing Yin, fortifying spleens, moistening lungs, and tonifying kidneys [2]. Thus, it has been considered to have various pharmacological effects, including promoting physical energy, improving gastrointestinal function, protecting respiratory system, improving sexual performance, and strengthening the immune system. Furthermore, PR can also be used to treat some symptoms such as fatigue, 
weakness, indigestion, inappetence, diabetes, cough, sexual dysfunction, backache, knee pain, and premature hair greying [3].Therefore, huangjing is frequently used in Chinese people's daily diets [4], and a variety of functional foods with huangjing as main raw material are supplied on the market [5].

However, huangjing must be processed through traditional Chinese processing technology to be used in traditional medicine and the food industry in China. The steaming technology, an effective method to change food from raw to mature, plays an important role in the fields of food manufacturing and food processing worldwide. It is well known that steaming is a traditional processing method for huangjing, especially the strategy of nine cycles of steaming and drying, which accounts for $56.6 \%$ of all ancient literatures on the processing methods of huangjing [6]. The steaming process can affect major quality parameters associated with food products, such as color, odor, taste, polysaccharides, volatile compounds, and secondary metabolites [7-10].

As a sort of popular tonic food in China, PR is often processed by repeated steaming and drying procedures until the rhizome turns black, soft, and sweet, which can significantly enhance its nourishing function and avoid throat irritation. Over the years, methods for determining the effective processing level of PR have been explored, while the steaming time, processing degree, and objective judgment indexes (e.g., color, texture, taste, and odor) of PR are still unclear. In addition, there are few reports on evaluations of the sensory quality of PR processed under different numbers of processing cycles.

Over the past few years, gas chromatography-mass spectroscopy (GC-MS), electronic nose (E-nose), and electronic tongue (E-tongue) have been increasingly applied in the food industry for quality control [10-13]. E-nose and E-tongue have provided support for the objective expression of the odor and taste of medicinal and edible homologous medicines. Many volatile compounds have been found to have several therapeutic properties, including antioxidation, antitumor, anti-inflammation, and antimicrobial activities [14]. Three terpenoids, i.e., $\alpha$-pinene, camphene, and $\beta$-caryophyllene, have been found to have the potential to treat a variety of inflammatory diseases such as respiratory inflammation, atopic dermatitis, arthritis, and neuroinflammation [15]. Geraniol has been found to possess various pharmacological properties, including antitumor, antioxidant, anti-inflammatory, antimicrobial, antidiabetic, cardioprotective, and neuroprotective activities [16,17]. Betacaryophyllene has been identified a $\mathrm{CB}_{2}$ receptor agonist with pharmacological activities such as antidiabetic, anti-inflammatory, and anticancer [18]. Various techniques have been used to extract volatile compounds in the field of herbal medicine, such as steam distillation, simultaneous distillation-extraction, static headspace, and headspace solid-phase microextraction (HS-SPME) [19-21]. In contrast to a liquid-liquid extraction method, static headspace is a non-destructive and non-invasive method that avoids the contamination of solvent impurities. As compared with HS-SPME, the static headspace is simple and low cost [22]. Therefore, static headspace is suitable for routine analysis and meets the criteria of green analytical chemistry principles. GC-MS can be used to reveal changes in volatile components during processing. Chemometric analysis is a simple strategy to identify traditional Chinese medicines, which has been widely used in quality control and evaluation of Chinese materia medica [23,24]. The chemometric methods of principal component analysis (PCA) and hierarchical cluster analysis (HCA) are commonly used in the field of herbal medicines for identification investigations, including species, geographical location, processing production, and harvesting time, as well as other factors that influence the quality of herbal materials [25]. GC-MS combined with chemometric analysis was recently used to analyze ginseng [26], goji berry [27], rhizomes of Curcuma [28], and Ephedrae herba [29]. Thus, modern analytical techniques integrated with chemometrics are becoming useful analytical tools for quality control of herbal medicines.

The objective of this study was to understand the influence of traditional Chinese processing on volatile profiles and flavor properties of PR samples. E-nose, E-tongue, and headspace GC-MS were applied to characterize volatile constituents and flavor properties in the nine processing cycles of PR. A chemometric analysis was conducted with the aim to 
accurately distinguish PR with different processing levels, and to screen out potential markers for the identification of processing production. The results are useful for monitoring the changes in sensory quality and identifying the processing level of PR samples.

\section{Results and Discussion}

\section{1. $p H$ Measurement}

The color of the samples gradually became darker after each processing cycle (Figure S1). Figure 1 shows that the $\mathrm{pH}$ value for $\mathrm{PR}$ samples decreased progressively as the processing level increased, undergoing dynamic changes from 5.50 (initial value) to 3.91. The decrease in $\mathrm{pH}$ could be due to the progression of the Maillard reaction and the generation of organic acids accompanied by a reduction in amino groups [30].

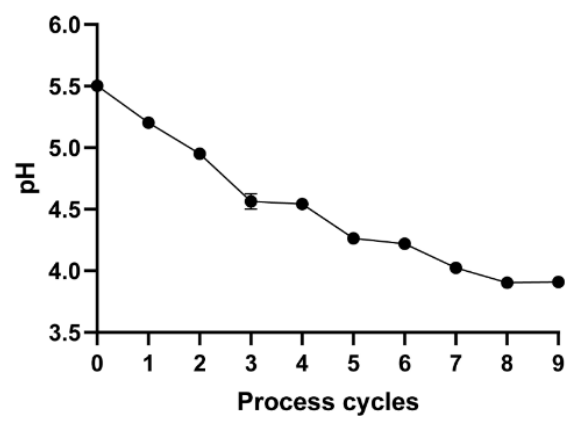

Figure 1. Changes in $\mathrm{pH}$ values for $\mathrm{PR}$ samples with different processing levels.

\subsection{Electronic Nose}

\subsubsection{Electronic Nose Response to Different Processing Levels of PR}

The typical E-nose responses to different processing levels is shown in the Supplementary Materials (Figure S2). A total of 10 PR samples with different processing levels were detected by E-nose, and the response curve is shown in Figure S2. The horizontal axis is the sampling time (s), and the vertical axis is the response signal value of the E-nose sensor (G/G0 or G0/G). Each curve represents the change in response intensity of a sensor during the sampling time. The response values conductivity ratio of seven sensors were G/G0, and the response values of the other three sensors, i.e., W1C, W3C, and W5C were G0/G, in which $G$ is the conductivity of the sensor after contacting the sample gas, and G0 is the conductivity of sensor cleaned by standard activated carbon filtered gas. The curve represents the response signal value of 10 metal oxide sensors to the odor of the sample (different colors represent different metal oxide sensors).

The data show that the response values of the 10 sensors of the E-nose gradually increased with an increase in acquisition time, and the curve gradually stabilized at $30 \mathrm{~s}$. When the change range of the curve was small, the sensitivity of the electronic nose to the odor had reached a stable state. As shown in Figure S2, stabilization was reached within 50-60 s. The W1W sensor was the most sensitive variable, and the W1W response value of the RPR sample was relatively low. With increased processing, the W1W response value gradually increased, and the fifth steamed sample (PPR5) stabilized.

\subsubsection{Data Analysis on Odor Characteristics of PR with Different Processing Levels}

According to the odor response value of different samples, an odor characteristic map of PR samples was established, which is shown in Figure 2. The circumference represents the name of the sensor, and the response value of different samples is displayed in polar coordinates by color patches. By using a one-way ANOVA test and Kruskal-Wallis test (Table S2), there were differences in the 10 aroma indicators of PR with different processing levels $(p<0.05)$. According to Figure 2 and sensor response values (Table S2), sensors W1W, W5S, W2W, and W1S were more sensitive to the odor of samples, and their response values varied significantly from the RPR sample to the PPR9 sample. The odor indicators 
of the 10 samples were different, which was further combined with the PCA unsupervised analysis method to analyze the odor characteristics of samples collected by E-nose to obtain the significant odor characteristics of PR samples with different processing levels.

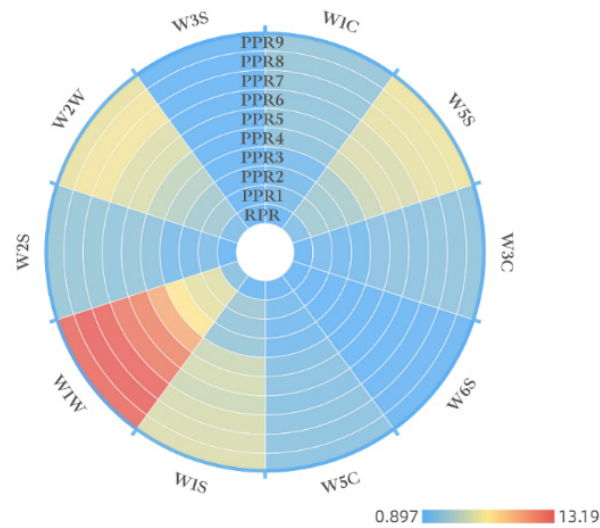

Figure 2. Odor characteristics of PR samples with different processing levels.

\subsubsection{Differentiation of Processed PR Samples by PCA}

To evaluate the influence of the steaming process on the grouping of PR, a PCA was performed on the dataset of the response values of E-nose samples. The biplots of score and loading of the processed samples are presented in Figure 3. The data showed that the grouping of the samples was from left to right along the $\mathrm{t} 1$ axis with increased processing levels. The RPR sample, first PR sample (PPR1), second PR sample (PPR2), and third PR sample (PPR3) were located on the left side of the score plot, while the fourth PR sample (PPR4), fifth PR sample (PPR5), sixth PR sample (PPR6), seventh PR sample (PPR7), eighth PR sample (PPR8), and ninth PR sample (PPR9) were located on the right side of the score plot, among which there was an overlap in the PPR5-PPR9 samples. It was preliminarily considered that the smell of the fifth PR sample was similar to that of the ninth PR sample.
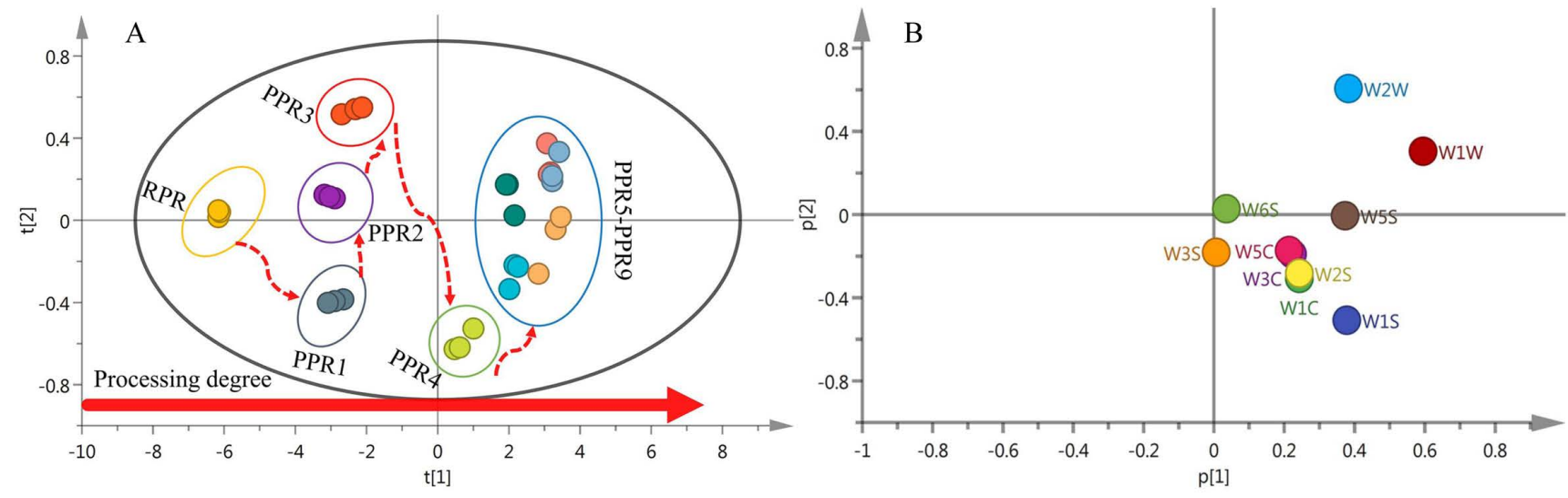

Figure 3. Principal component analysis PCA biplots for 10 different processed PR samples based on E-nose response data: (A) Score plot; (B) loading plot.

The cumulative variance contribution R2X of the first three principal components reached 0.997 , and the predictive ability parameter Q2 was 0.984 , which indicated that the PCA model was a well-fitted and predictive model. Principal component factor loading analysis showed the contribution rate of each factor variable to the principal component. The greater the absolute value of the load eigenvector, the greater the contribution to the principal component. The principal component factor loading matrix is presented in Table 1. The first principal component (PC1) had the largest amount of information, and the independent variance explanation contribution reached 0.984 . The absolute values of 
the eigenvectors of sensors W1W (0.596), W1S (0.379), and W5S (0.375) were larger than the remaining sensors. In the second principal component (PC2), the absolute values of the eigenvectors of sensors W2W $(0.610)$ and W1C $(-0.307)$ were relatively large. In addition, in the third principal component (PC3), the absolute values of the eigenvectors of sensors W3S (0.882) and W6S (0.304) were relatively large. Therefore, the PC1 mainly depended on sensors W1W, W1S, and W5S; the PC2 depended on sensors W2W and W1C; and the PC3 depended on sensors W3S and W6S.

Table 1. Principal component factor loading for E-nose data.

\begin{tabular}{cccc}
\hline Sensor & PC1 & PC2 & PC3 \\
\hline W1C & 0.242 & -0.307 & -0.059 \\
W5S & 0.375 & -0.002 & 0.166 \\
W3C & 0.224 & -0.192 & -0.025 \\
W6S & 0.035 & 0.028 & 0.304 \\
W5C & 0.212 & -0.172 & 0.004 \\
W1S & 0.379 & -0.511 & -0.290 \\
W1W & 0.596 & 0.308 & 0.054 \\
W2S & 0.245 & -0.279 & 0.103 \\
W2W & 0.381 & 0.610 & -0.014 \\
W3S & 0.005 & -0.179 & 0.882 \\
R2X & 0.984 & 0.0104 & 0.00277 \\
R2X (cum) & 0.984 & 0.995 & 0.997
\end{tabular}

Figure 3B shows that the factor loading corresponding to the characteristic values of different sensors was mainly distributed along the $\mathrm{p} 1$ axis on the right side of the loading plot, and some overlap was observed. Sensors W1W, W2W, W1S, and W5S significantly contributed to the differentiation of PR samples. The characteristic values of sensors with high overlap were highly similar. In combination with Table 1, the absolute values of the eigenvectors of the three partially overlapped sensors $\mathrm{W} 3 \mathrm{C}, \mathrm{W} 5 \mathrm{C}$, and W2S were small, causing little contribution to the grouping of the samples, and therefore redundant information could be eliminated.

The results of the PCA analysis showed that the number of steaming and drying cycles had a significant effect on the odor characteristics of PR samples. The significant odor differences between the RPR sample and the PR samples were W1W, W2W, W1S, and W5S. After the fifth processing cycle, the odor index basically did not change, and the samples from PPR5 to PPR9 were pooled.

\subsection{Electronic Tongue}

\subsubsection{Data Analysis on Taste Characteristics of Different Processed PR Samples}

According to the taste response value of different samples, the taste characteristic map of PR samples is presented in Figure 4. The characteristic map of E-tongue was similar to that of E-nose, which presented the taste difference of the samples. Using the one-way ANOVA test and Kruskal-Wallis test, it is shown in Table S3 that there were differences in the nine taste indicators of PR with different processing levels $(p<0.05)$. According to the data presented in Figure 4 and taste sensor response values (Table S3), the taste differences of 10 different samples were mainly reflected in the indexes of sourness, sweetness, astringency, aftertaste-astringency, umami, and richness. With an increased degree of processing, the sourness index increased significantly. However, the sweetness decreased, the astringency and aftertaste-astringency increased, the umami taste decreased, and the richness increased. 


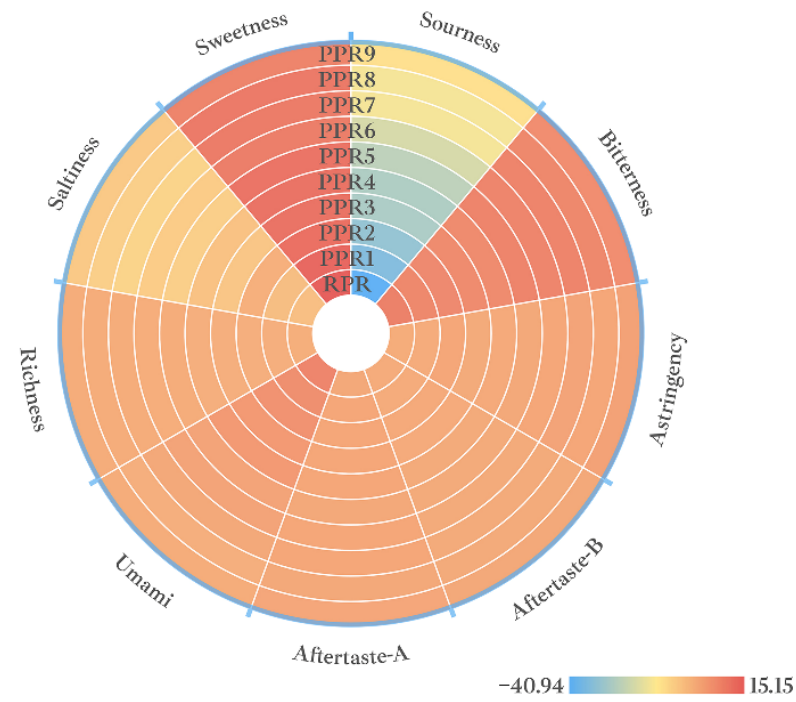

Figure 4. Taste characteristics of PR samples with different processing levels.

\subsubsection{Differentiation in Processed PR Samples by PCA}

To distinguish PR samples from different processing levels, a PCA was also performed on the E-tongue dataset, taking the response values of taste sensors as input variables. Figure 5A shows that the RPR, PPR1, PPR2, PPR3, and PPR4 samples were located on the left side of the score plot, whereas the PPR5, PPR6, PPR7, PPR8, and PPR9 samples were located on the right side of the score plot, in which there was an overlap in the PPR5-PPR8 samples. It was preliminarily considered that the taste from the fifth PR sample to the eighth PR sample was similar.
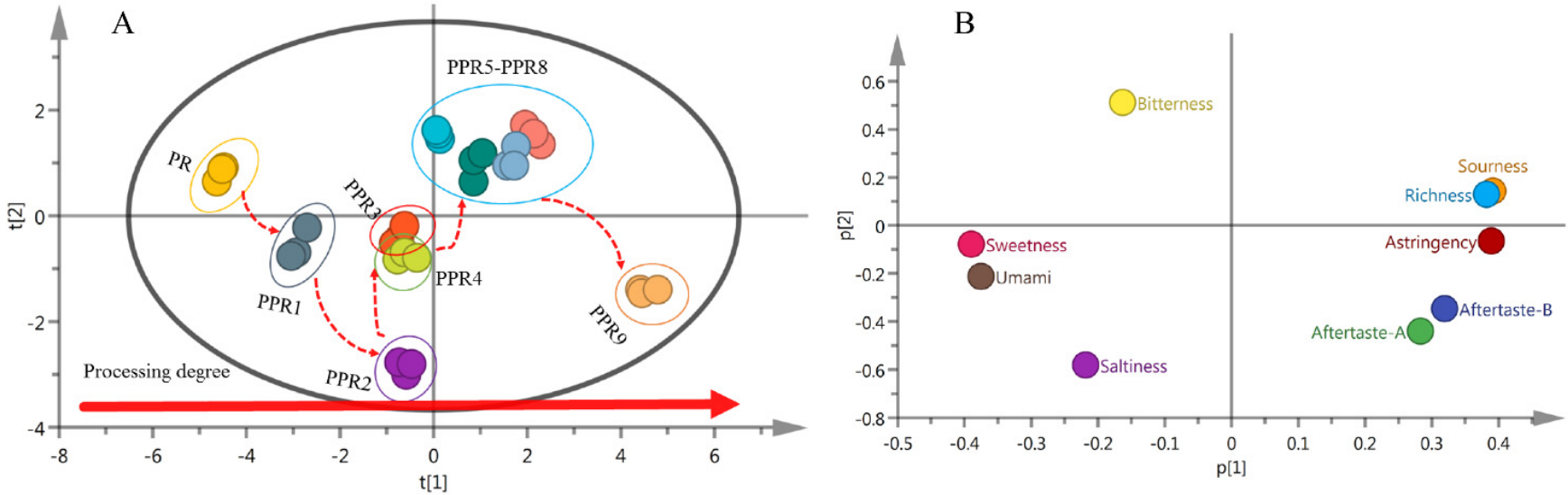

Figure 5. Principal component analysis PCA biplots for 10 different processed PR samples based on E-tongue response data: (A) Score plot; (B) loading plot.

The cumulative variance contribution $\mathrm{R} 2 \mathrm{X}$ of the first three principal components reached 0.956 , and the predictive ability parameter Q2 was 0.847 , which indicated that the PCA model had good fitting and prediction properties. The taste index corresponding to each sensor of the E-tongue was regarded to be a factor variable, and the loading analysis of principal component factor was performed. The principal component factor loading matrix is shown in Table 2. The PC1 gave the most information; the independent variance explanation contribution reached 0.682 , and the absolute values of the eigenvectors of the taste indexes for sourness (0.391), astringency (0.390), sweetness $(-0.390)$, richness $(0.382)$, umami $(-0.376)$ were large. The taste indexes for saltiness $(-0.579)$ and aftertaste-A $(-0.438)$ in the second principal component had large eigenvector absolute values, and the taste indexes for bitterness (0.772) and aftertaste-B (0.443) in the third principal component 
had high absolute values. The data showed that the PC1 mainly depended on the taste indexes for sourness, astringency, sweetness, richness, and umami, the second principal component depended on the taste indexes for saltiness and aftertaste-A, and the third principal component depended on the taste indexes for bitterness and aftertaste-B.

Table 2. Principal component factor loading for E-tongue data.

\begin{tabular}{cccc}
\hline Taste & PC1 & PC2 & PC3 \\
\hline Sourness & 0.391 & 0.146 & -0.126 \\
Bitterness & -0.163 & 0.512 & 0.772 \\
Astringency & 0.390 & -0.065 & 0.228 \\
Aftertaste-B & 0.319 & -0.344 & 0.443 \\
Aftertaste-A & 0.282 & -0.438 & 0.135 \\
Umami & -0.376 & -0.209 & 0.197 \\
Richness & 0.382 & 0.131 & 0.169 \\
Saltiness & -0.219 & -0.579 & 0.214 \\
Sweetness & -0.390 & -0.077 & 0.093 \\
R2X & 0.682 & 0.216 & 0.058 \\
R2X (cum) & 0.682 & 0.899 & 0.956 \\
\hline
\end{tabular}

Figure 5B shows that the taste indexes for sourness, richness, astringency, sweetness, and umami had a greater contribution to the classification of PR samples with different processing levels. Moreover, Table S3 shows that the taste characteristics of RPR were mainly sweet and umami, and the taste characteristics of the samples from PPR5 to PPR9 were sourness, richness, and astringency.

The results of the PCA analysis showed that the number of steaming and drying cycles had a significant effect on the taste characteristics of PR samples. Furthermore, the taste index did not significantly change after the fifth processing cycle.

The minimum detectable value of the taste sensor of the SA402B electronic tongue was a $20 \%$ change in concentration, and the response output value could be converted into "taste information", thereby, indicating that one unit of the response value of the taste sensor represented the taste difference caused by a $20 \%$ concentration difference in the sample, which was also the smallest unit of taste difference that people began to perceive. The full range of taste perception is 25 units, and the range of taste response that was experienced is acid (-13-12), bitter (0-25), astringency (0-25), salty (-6-19), and sweet (0-25) [31]. Table 3 shows that among the nine taste indicators, the sweetness values of the 10 samples of PR with different processing levels ranged from 8.62 to 15.15 , which accounted for a large portion of the sweetness range perceived by humans; only the sour taste value of the ninth PR sample (PPR9) was in the range of human perception, which was experienced. In addition, other taste indexes accounted for a small proportion of human perception. Therefore, what people really experienced before and after the steaming process was that there was always sweetness, and there was sourness only in the ninth PR sample.

Through literature studies, we found that the sample solution of traditional Chinese medicine measured by electronic tongue included water-extracted solutions [32,33]. In Chinese clinical application, PR is mostly taken after water decoction. For the PR samples measured by the E-tongue, to achieve taste that was closer to clinical application, the experimental samples were prepared using a water extraction method. 
Table 3. Key flavor components in PR samples with different processing levels.

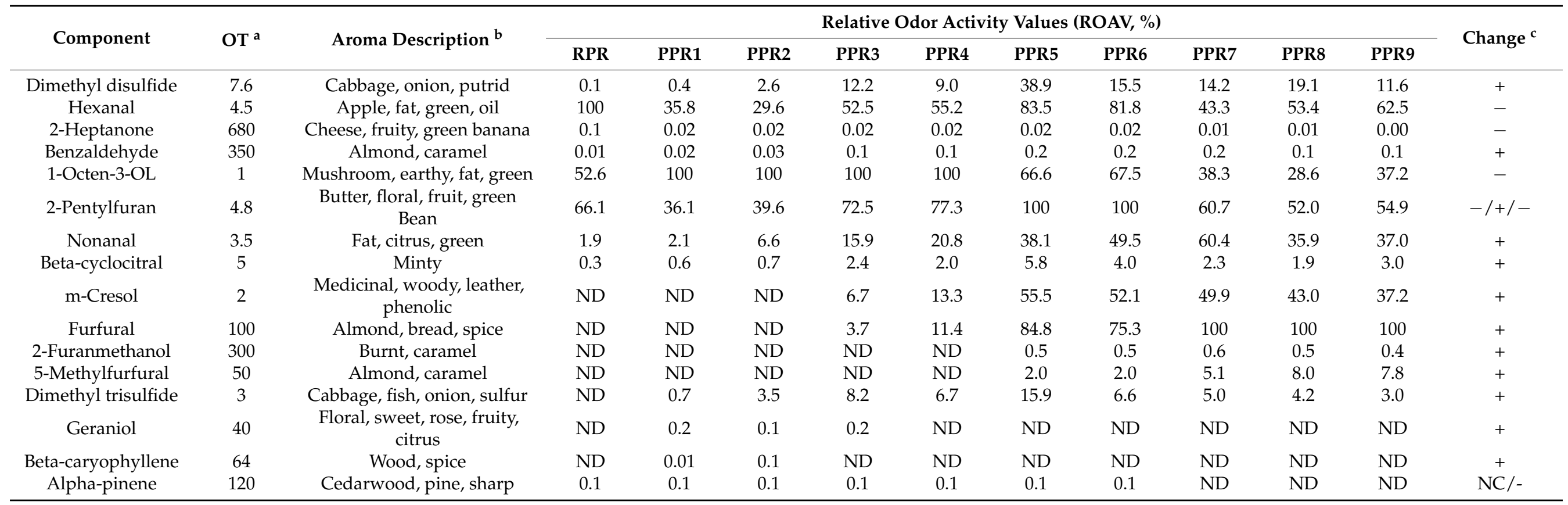

a Odor threshold (OT) in ppb. ${ }^{b}$ Aroma descriptors from online databases: FEMA (http:/ / www.femaflavor.org, accessed on 10 July 2021) and Flavornet (http:/ / www.flavornet.oro accessed on 10 July 2021). ${ }^{c}$ Direction of change in ROAV for RPR vs. other processed PR samples: decrease (-), increase (+), or no change (NC). Only flavor compounds with ROAV $\geq 0.1$ at least in PR samples are presented. ND, non-detectable. 


\subsection{Headspace GC-MS}

2.4.1. Volatile Compounds in the Different Samples by Headspace GC-MS

To evaluate the effect of the traditional steaming process on the flavor of PR samples, HS-GC-MS was used to analyze the volatile compounds of each sample afforded by different processing levels. The total ion chromatograms of all samples with different processing levels are shown in Figure 6A. The main peak appeared at $5.3 \mathrm{~min}$, and its ionic strength decreased significantly with an increase in the number of processing cycles. Two compounds, hexanal and octane, were identified from the main peak. Hexanal had a lower odor threshold as compared with octane. It showed that $n$-hexanal could be used as a component marker to distinguish samples with different processing levels. The individual chromatograms of 10 samples are shown in Figure S3. The number and relative content of volatile compounds from each sample are shown in Figure 6, and additional details are presented in Tables S4 and S5 in the Supplementary Materials.

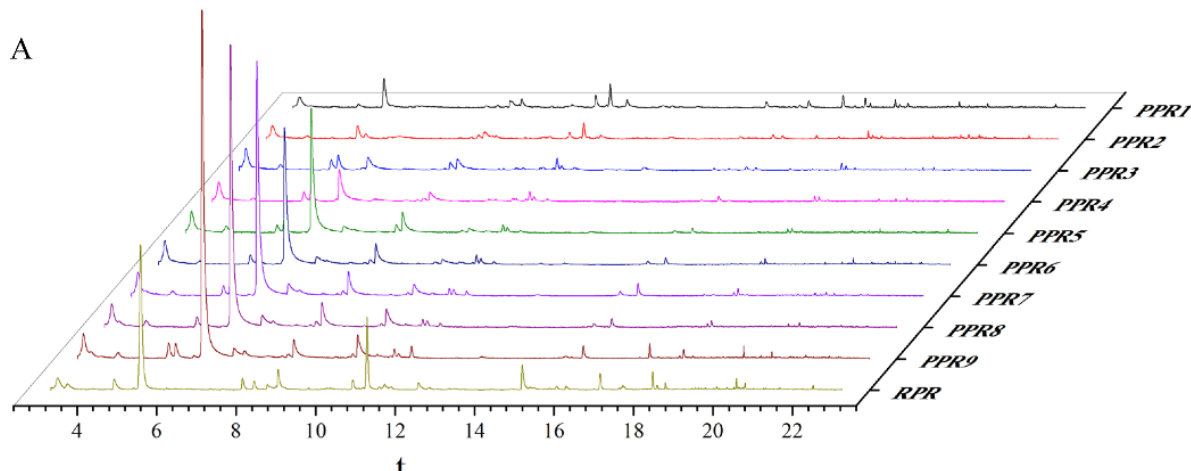

B

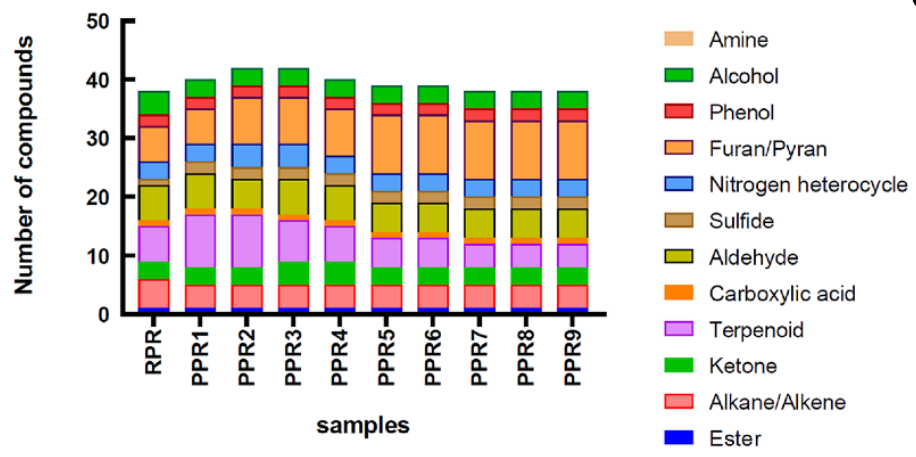

$\mathrm{C}$

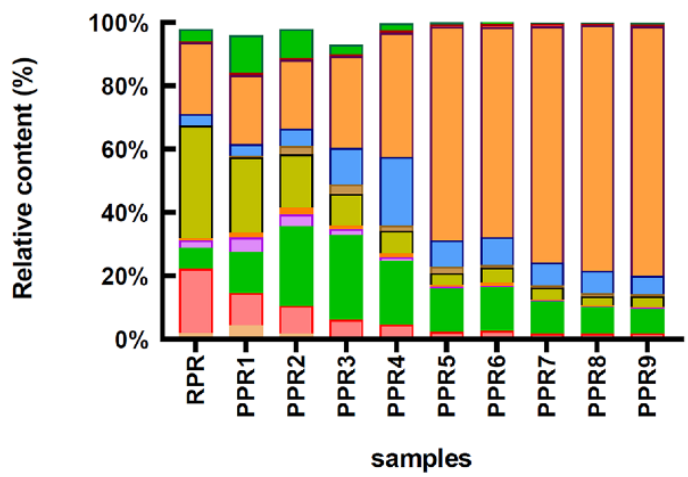

Figure 6. Volatile compounds of PR samples with different processing levels: (A) Total ion chromatograms; (B) number of volatile compounds; (C) relative content of volatile compounds.

A total number of 54 volatile compounds were detected in all PR samples, which were classified as follows: 4 alcohols, 4 phenols, 7 aldehydes, 9 terpenoids, 4 ketones, 1 ester, 12 furans/pyrans, 1 carboxylic acid, 5 alkanes/alkenes, 4 nitrogen heterocycles, 1 amine, and 2 sulfides. The most abundantly found flavor families were furans/pyrans, terpenoids, and aldehydes (Figure 6B).

The relative contents of the main volatile compounds in RPR in decreasing order were as follows: aldehydes $(35.56 \%)>$ furans/pyrans $(22.60 \%)>$ alkanes/alkenes $(22.16 \%)>$ ketones $(6.45 \%)$. After the steaming and drying cycle, the relative contents of volatile compounds in the PPR9 sample were furans/pyrans $(78.76 \%)>$ ketones $(7.81 \%)>$ nitrogen heterocycles $(5.90 \%)>$ aldehydes $(3.30 \%)>$ alkanes/alkenes $(1.73 \%)$. These findings showed that as compared with the RPR sample, the total relative content of furans/pyrans was significantly increased, while aldehydes and alkanes/alkenes were decreased. The relative content of furans/pyrans in the PPR9 sample reached $78.76 \%$, which increased by $56.16 \%$ as com- 
pared with that of the RPR sample (22.60\%). The total relative contents of aldehydes and alkanes/alkenes in the PPR9 sample were $3.30 \%$ and $1.73 \%$, respectively, which decreased by $32.26 \%$ and $20.43 \%$ as compared with that in the RPR sample (35.56\% and $22.16 \%$ ). With an increase in the number of cycles, alcohols, terpenoids, ketones, sulfides, and nitrogen heterocycles first increased, then decreased, and then stabilized, but no significant changes were observed for esters, carboxylic acids, and phenols (Figure 6C).

As shown in Table S6, there were 26 common compounds in all samples. With an increase in processing cycles, common volatile compounds were significantly decreased. As compared with RPR, 15 compounds were newly produced in processed samples (shown in Table S7), including 6 furans (2,5-dimethylfuran, furfural, 2,5-furandione, 2-furanmethanol, 2-ethyl-5-methylfuran, and 5-methylfurfural), 3 terpenoids (geraniol, (+)-alpha-muurolene, and beta-caryophyllene), 2 phenols (m-cresol and butylated hydroxytoluene), 1 ketone (cyclohexanone), 1 aldehyde (2-ethyl-2-hexenal), 1 nitrogen heterocycles (1,4-dimethylpyrazole), and 1 sulfide (dimethyl trisulfide). These data were in accordance with data presented in the literature [34-36]. As shown in Table S8, 13 volatile components could not be gradually detected, of which 3 chemical components were unique to RPR, namely 3-methyl-1-pentanol, undecane, and gamma-undecalactone. The difference between the contents and types of volatile compounds present determine the unique aroma profile of each sample.

In previous studies, it has been shown that aldehydes were relatively abundant headspace volatiles and had a very low odor threshold, therefore, they played a significant role in the characteristic flavor of food [37]. Aldehydes were found to be the major class of volatile compounds in all samples. Most aldehydes have been reported to be highly associated with almond, green, and citrus aromas [8,38]. Hexanal made a significant contribution to the aldehydes in RPR (29.88\%). As a flavor active compound, hexanal conferred a rancid smell at high amounts, while in low content it produced a pleasant green aroma [39]. Hexanal decreased as the processing cycle increased, whereas nonanal (citrus, green), benzaldehyde (almond aroma), and beta-cyclocitral (minty) increased.

The content of alkanes/alkenes significantly decreased with increased processing cycles. The RPR sample had the highest ratio of alkanes/alkenes, with the most abundant octane, which accounted for $21.09 \%$ of the alkanes/alkenes class. Generally, alkanes/alkenes did not significantly contribute to the aroma due to their high odor threshold and they had an odor similar to gasoline [40].

The content trend of ketone showed that it increased greatly and rapidly in the early stage, and then decreased and stabilized in the later stage. The occurrence of ketone compounds usually endowed food with a cheese, fruit, and caramel flavor [41].

The alcohols detected in all samples were mainly 1-octen-3-ol, 2-ethylhexanol, and 2-methylcyclohexanol, which commonly produced mushroom, earthy, green, rose, and citrus aromas. The PPR1 sample reached the highest alcohol content of $11.90 \%$, with the most abundant 1-octen-3-ol, which was responsible for the mushroom-like odor.

Furan/pyran is a class of oxygenated heterocyclic compounds for food flavors. Because the furan ring is more stable than the pyran ring, more furan is produced during the pyrolysis process. Furans were detected in all samples, which were mainly produced during Maillard reactions and thermal degradation of sugars [42]. The processed samples contained abundant furans, which increased rapidly with increased processing cycles. Among furans, furfural was the dominant compound in the post-processing stage, which contributed to the flavors of almond, bread, burnt, and spicy in food.

RPR had the characteristics of a "long-time smell of raw flavor, and a dazzling feeling" [34]. However, hexanal has been shown to be the main component of indoor environmental irritants, and was irritating to eyes, the respiratory system, and the skin $[43,44]$. The presence of undecane can also cause damage to the central nervous system, respiratory irritation, and even chemical pneumonitis [45]. The content of hexanal was the highest in RPR, and undecane was a unique component in RPR. Hexanal and undecane might be 
volatile components that can cause eye irritation, but further experiments are needed to prove this.

According to the literature [46,47], key-aroma compounds have been identified through calculation of their relative odor activity values (ROAV). Compounds with ROAV $\geq 1$ were considered to be key flavor components that significantly contributed to the final aroma profile, and compounds with $0.1 \leq \mathrm{ROAV}<1$ had an important modifying effect on the overall flavor of the sample. All the compounds with ROAV $\geq 0.1$ are listed in Table 3 . Thus, a total of 16 compounds were found to effectively contribute to the final flavor profile of the PR samples. Among them, four volatiles were observed to be key volatiles ( $R O A V \geq 1)$ in the RPR and PPR1 samples, six volatiles were key volatiles in the PPR2 sample, and nine volatiles were key volatiles in the PPR3 and PPR4 samples. In the PPR5-PPR9 samples, 10 volatiles were key volatiles. The data showed that repeated steaming and drying was conducive to the diversity of the flavor of PR samples. The types of key flavor compounds did not significantly change after the fifth processing cycle.

The heatmap of key flavor components of PR samples with different processing levels is shown in Figure 7. Analysis of the relative amounts of 16 key volatiles in each processing level showed that the concentration of hexanal, 1-octen-3-ol, and 2-pentylfuran were significantly high in all the PR samples. The PPR1 and PPR2 samples showed a similar or improved composition of volatile compounds as compared with the RPR sample. Furthermore, the PPR3 and PPR4 samples exhibited a similar flavor chemical composition. The samples from PPR5 to PPR9 also exhibited similar compositions, indicating the flavor reached a steady state after four cycles.

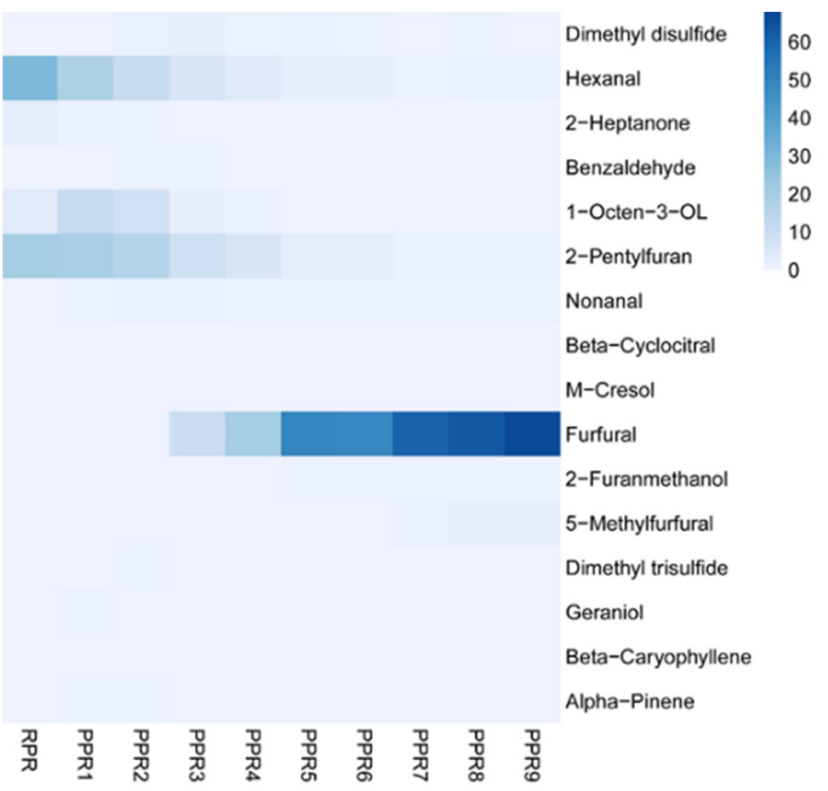

Figure 7. Heatmap of key flavor components of PR samples with different processing levels. The colors from white to blue represent the relative amounts of the flavor components from low to high.

In general, volatile markers associated with grassy, green, gasoline-like, and irritation, (e.g., hexanal and undecane) decreased during the processing cycle, whereas thermal-load, cabbage (e.g., dimethyl disulfide and dimethyl trisulfide), browning, almond, and burnt (e.g., furfural) indicator compounds increased during the processing cycle. Due to the abovementioned findings, PPR was more suitable for food applications with desirable aromas than RPR. It was useful for the determination of processing level in food and pharmaceutical industries based on samples with specific flavor characteristics. 


\subsubsection{Differentiation of Ten Different Processed PR Samples by PCA}

A PCA was performed on the data matrix (30 samples $\times 12$ volatile classes) to observe a possible sample distribution according to different processing levels. The cumulative variance contribution $\mathrm{R} 2 \mathrm{X}$ of the first three principal components reached 0.971 , and the predictive ability parameter Q2 was 0.836 . As shown in the score plot of PCA (Figure 8A), the samples exhibited a tendency to form three major groups. The RPR and PPR1 samples were all located in the third quadrant of the graph, while the PPR2, PPR3, and PPR4 samples were located in the second quadrant. The PPR5 and PPR6 samples were distributed close to the fourth quadrant, whereas the PPR7, PPR8 and PPR9 samples were located in the fourth quadrant of the graph. According to the PCA loading plot (Figure 8B), aldehydes, alkanes/alkenes, ketones, nitrogen heterocycles, and furans/pyrans had a greater influence on the differentiation ability as compared with other components. The aldehydes and alkanes/alkenes mainly distinguished RPR and PPR1 samples from other processed samples. In addition, ketones and nitrogen heterocycles contributed more to the PPR3 and PPR4 samples. Finally, the contents of furans/pyrans correlated more with the PPR5, PPR6, PPR7, PPR8, and PPR9 samples.
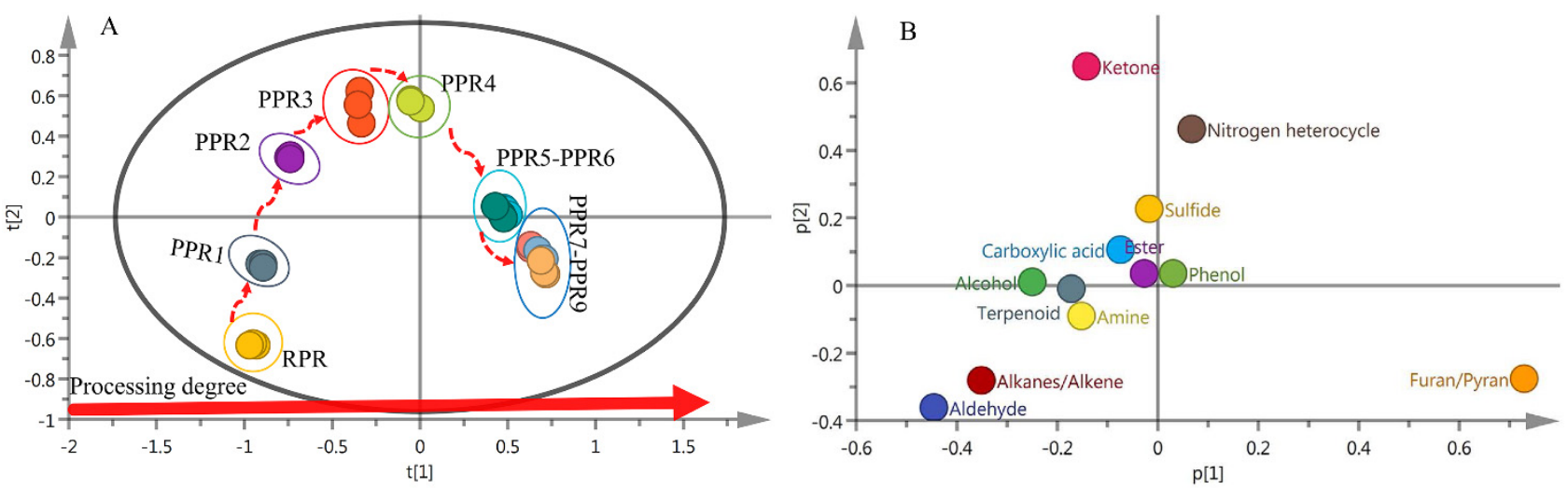

Figure 8. Principal component analysis PCA biplots for 10 different processed PR samples based on GC-MS data: (A) Score plot; (B) loading plot.

\subsection{Correlation Analysis of $\mathrm{pH}$ and E-Tongue}

A Pearson correlation analysis was conducted to determine the relationship between $\mathrm{pH}$ and $\mathrm{E}$-tongue sensors (Figure $9 \mathrm{~A}$ ). The $\mathrm{pH}$ value showed strong positive correlations with the sweetness and umami of the RPR sample, while $\mathrm{pH}$ value showed strong negative correlations with the sourness and richness of the PPR9 sample. The result indicated that $\mathrm{pH}$ value could discriminate PR samples by responding to human-perceived taste indicators such as sweetness and sourness.

\subsection{Correlation Analysis of E-Nose and E-Tongue}

The flavor of PR samples was developed through a series of complex processes. Taste was affected by the perception of odor, and its changes could be partially explained by changes in smell. Figure 9B shows that E-nose sensors were associated with E-tongue sensors, and changes in the taste would be further reflected in the changes of odor. Moreover, it was revealed that E-nose had the feasibility of evaluating taste-presenting substances and achieving rapid and non-destructive prediction of sample taste. 
A

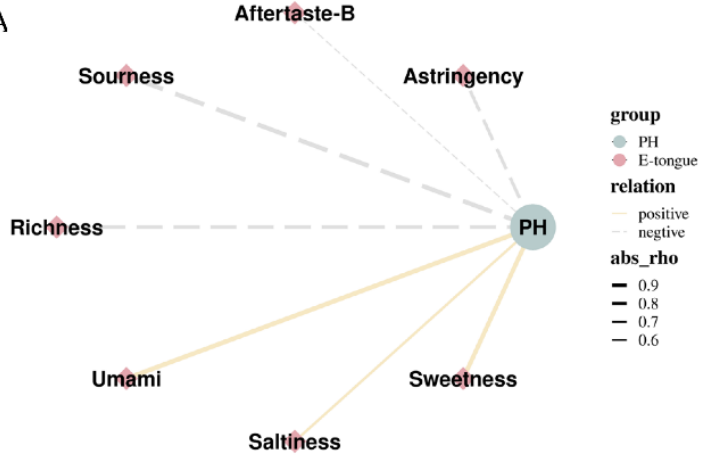

$\mathrm{B}$

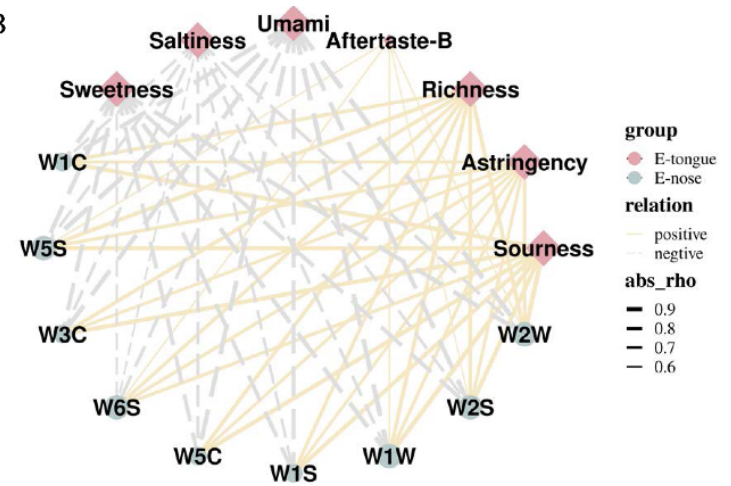

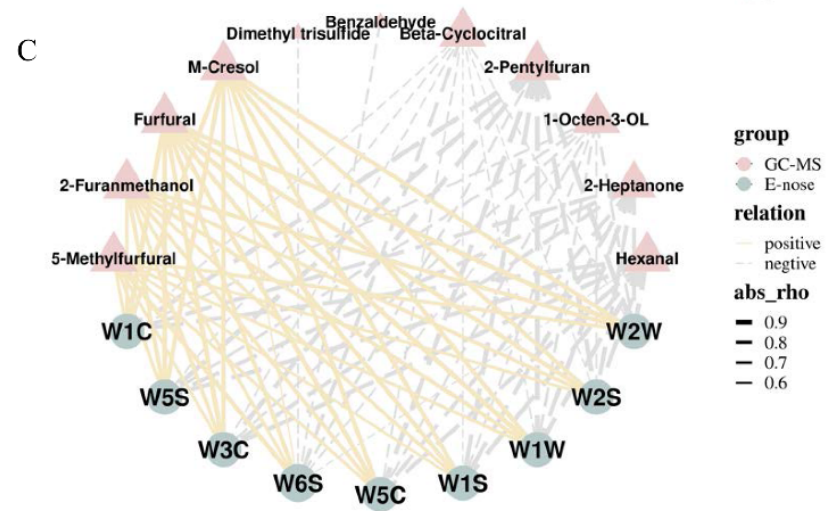

Figure 9. Correlations among $\mathrm{pH}$, E-nose, E-tongue, GC-MS for 10 different processed PR samples: (A) Between $\mathrm{pH}$ value and E-tongue data; (B) between E-nose data and E-tongue data; (C) between E-nose data and GC-MS data.

\subsection{Correlation Analysis of E-Nose and GC-MS}

Sixteen volatile compounds with high relative odor activity values (ROAV $\geq 0.1)$ based on GC-MS analysis were selected to correlate with E-nose signals (Figure 9C). The results showed that the signal intensities of E-nose sensors had significant and positive correlations with the abundances of m-cresol, furfural, 2-furanmethanol, and 5-methylfurfural, which indicated that E-nose sensors were sensitive to furan derivatives and phenolic compounds. In comparison, the intensities of E-nose signals were negatively correlated with the abundances of hexanal, 2-heptanone, 1-octen-3-ol, and 2-pentylfuran. Therefore, these eight volatiles might be related significantly to the flavor of the PR samples. This showed that E-nose was capable of distinguishing PR samples by responding specifically to volatile compounds.

\subsection{HCA of the Fusion Dataset of $p H, E-N o s e, E-T o n g u e$, and GC-MS}

A Cluster analysis was performed by using the between-groups linkage method and the squared Euclidean distance to the fused dataset comprised of $\mathrm{pH}, \mathrm{E}-$ nose, E-tongue, and HS-GC-MS data. The dendrogram, shown in Figure 10, indicates that the relationships and distribution among the samples in different processing levels and four main clusters of the samples were as follows: RPR (Cluster 1); PPR1, PPR2, PPR3, and PPR4 (Cluster 2); PPR5, PPR6, PPR7, and PPR8 (Cluster 3); and PPR9 (Cluster 4). These classification groups were consistent with the PCA results in which all samples were fully distinguished according to their processing levels. 


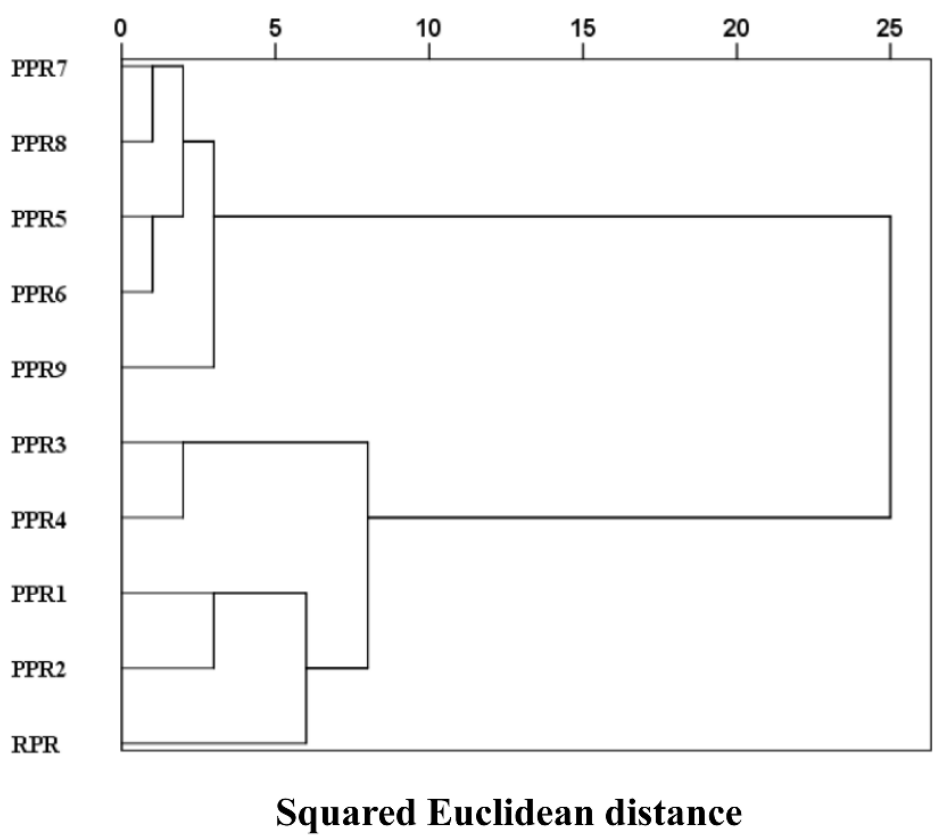

Figure 10. Dendrograms of HCA with a dataset including $\mathrm{pH}, \mathrm{E}-$-nose, E-tongue, and GC-MS data.

\section{Materials and Methods}

\subsection{Samples and Sample Preparation}

Fresh rhizomes of Polygonatum sibiricum (FPR) were harvested in October 2019 from Qingyuan County, Liaoning Province, one of the most famous PR growing regions in China; only fresh undamaged rhizomes with similar weight and size were selected for this study. There were 3 batches of samples, and the same batch of FPR was divided into 10 groups. According to the Chinese Pharmacopoeia (2020 edition), one group of FPR was processed into raw rhizomes of Polygonatum sibiricum (RPR), by boiling in water for 5 min, and then drying at $55^{\circ} \mathrm{C}$ for $9 \mathrm{~h}$. The remaining groups were subsequently processed into processed rhizomes of Polygonatum sibiricum samples (PPR1-PPR9), by $3 \mathrm{~h}$ of steaming (over boiling water), $1 \mathrm{~h}$ of simmering, followed by $9 \mathrm{~h}$ of oven drying $\left(55^{\circ} \mathrm{C}\right)$, repeated for $1-9$ cycles.

\section{2. $p H$ Measurement}

The $\mathrm{pH}$ value was determined using a Mettler Toledo FE $28 \mathrm{pH}$ meter (Mettler Toledo Instruments Co. Ltd., Shanghai, China) at room temperature. Ten-gram sample thick slices (range 2-4 mm) were soaked in 10 times the volume of distilled water for $1 \mathrm{~h}$ at room temperature $\left(25^{\circ} \mathrm{C}\right)$, and then refluxed twice by boiling for $1.5 \mathrm{~h}$ each time. Each extract was filtered, and the combined extracts were used for $\mathrm{pH}$ measurement.

\subsection{Electronic Nose Analysis}

The E-nose analyses were performed using a commercial PEN3 electronic nose (Airsense Analytics GmbH., Schwerin, Germany). The instrument consists of a gas-rate control system, a sensor array, and a pattern analysis software (WinMuster, v.1.6., Airsense Analytics GmbH., Schwerin, Germany). The sensor array is composed of 10 metal-oxide semiconductor sensors, i.e., W1C, W5S, W3C, W6S, W5C, W1S, W1W, W2S, W2W, and W3S, which are sensitive to specific volatile compounds (Table S1).

In brief, a sample of $4.0 \mathrm{~g}$ of powder ( $40 \mathrm{mesh}$ ) was put into a $250 \mathrm{~mL}$ beaker sealed with cling film and incubated for $30 \mathrm{~min}$ at $25^{\circ} \mathrm{C}$ to reach the headspace equilibrium. The test parameters were set as follows: the flow rate was $200 \mathrm{~mL} / \mathrm{min}$, measurement time $60 \mathrm{~s}$, and flush time $120 \mathrm{~s}$. Responses of the sensors were expressed as the ratio of conductance G/G0 (G and G0, respectively, indicate the conductance of the sensors in contact with sample gas and clean gas), which varied with different substances. Each sample was 
measured in triplicate and the average of sensor responses after stabilization was taken for subsequent analysis.

\subsection{Electronic Tongue Analysis}

The difference in taste of 10 samples was evaluated by using an electronic tongue (taste sensing system SA402B, Intelligent Sensor Technology Co., Kanagawa, Japan), which contained eight multichannel lipid membrane sensors: CA0, CT0, AAE, C00, AE1, GL1, $\mathrm{AC} 0$, and AE1(aftertaste), which were potentiometric sensors with specific sensitivity and selectivity to different taste substances.

A total of $1 \mathrm{~g}$ of dried sample powder ( 40 mesh) was extracted with $100 \mathrm{~mL}$ distilled water under ultrasonication $(40 \mathrm{kHz})$ for $30 \mathrm{~min}$. Subsequently, the solution was centrifuged at $3000 \mathrm{rpm}$ for $10 \mathrm{~min}$ at $25^{\circ} \mathrm{C}$ and the supernatant was obtained. The supernatant of each sample measured $80 \mathrm{~mL}$, was divided into two parts, and placed in two rows of circular sample cups in parallel. Acidity, bitterness, astringency, saltiness, sweetness, umami, and aftertaste were tested at room temperature $\left(25^{\circ} \mathrm{C}\right)$. Each sample was measured in quadruplicate. The data of the first cycle was removed, and the data of the following three cycles were taken as the measured values. After each measurement, the sensors were cleaned automatically.

\subsection{Headspace GC-MS Analysis}

The headspace autosampler combined with GC-MS was conducted to analyze the volatile compounds of PR samples. In brief, accurately weighed $3.0 \mathrm{~g}$ of different sample powder (40 mesh), was put into $20 \mathrm{~mL}$ headspace vials and capped with a PTFE/silicon septum. After incubation at $120^{\circ} \mathrm{C}$ for $20 \mathrm{~min}, 10 \mu \mathrm{L}$ of headspace gas was injected into to a gas chromatography inlet. The GC-MS analyses consisted of a TRACE 1300 Series GC and an ISQ Series MS (Thermo Fisher Scientific, Waltham, MA, USA). The volatiles were separated on a TG-5MS capillary column $(30 \mathrm{~m} \times 0.25 \mathrm{~mm}, 0.25 \mu \mathrm{m}$, Thermo Fisher Scientific, Waltham, MA, USA) based on the following analytical conditions: the injection port temperature was set to $250^{\circ} \mathrm{C}$, the flow rate of helium carrier gas was set to $1 \mathrm{~mL} / \mathrm{min}$, and the split ratio was 1:5. The condition of the oven temperature ramp was as follows: initial temperature of $35^{\circ} \mathrm{C}$ for $2 \mathrm{~min}, 5^{\circ} \mathrm{C} / \mathrm{min}$ to $75^{\circ} \mathrm{C}$ for $4 \mathrm{~min}, 15^{\circ} \mathrm{C} / \mathrm{min}$ to $95^{\circ} \mathrm{C}$ for $1 \mathrm{~min}$, and $20^{\circ} \mathrm{C} / \mathrm{min}$ to $150{ }^{\circ} \mathrm{C}$, followed by $15{ }^{\circ} \mathrm{C} / \mathrm{min}$ to $270{ }^{\circ} \mathrm{C}$ (maintained for $3 \mathrm{~min}$ ). The programmed temperature mode was carried out in the range from $35^{\circ} \mathrm{C}$ to $270{ }^{\circ} \mathrm{C}$. Mass spectra were acquired in electron impact (EI) mode at $70 \mathrm{eV}$, with the ion source temperature of $280^{\circ} \mathrm{C}$, and a transfer line temperature of $280^{\circ} \mathrm{C}$. The detection was performed in full scan mode over a mass range of $40-250 \mathrm{~m} / \mathrm{z}$, with a scan time of $0.2 \mathrm{~s}$ per scan.

First, the obtained GC/MS data were converted to an Analysis Base File (ABF) format using an ABF converter (https:/ / www.reifycs.com/AbfConverter/index.html, accessed on 8 April 2021), and then imported into the MS-DIAL software for deconvolution, peak detection, alignment, and filtering. The identification of volatile compounds was performed using MoNA mass spectral libraries and Kovats retention index (RI), as shown in Table S9, Figures S4 and S5. The results with dot and reverse dot product scores greater than 0.7 were selected. The retention indices of the identified compounds were calculated from the van den Dool and Kratz equation using the retention times of the alkane standards (C6-C20).The relative contents of components in each sample were determined by the normalization method $[11,48]$.

\subsection{Statistical Analysis}

To differentiate and classify samples, in this study, unsupervised principal component analysis (PCA) was performed on the data, using SIMCA software (version 14.1, Umeå, Sweden). The statistical significance test was performed by one-way ANOVA and the Kruskal-Wallis test using SPSS 25.0 software (SPSS Inc., Chicago, IL, USA). For statistical testing, $p<0.05$ was considered to be the critical level of significance. The hierarchical 
cluster analysis (HCA) was conducted using SPSS 25.0 software. The sensor response data were plotted to a polar coordinate heat map using the dycharts website (https: / Www. dycharts.com, accessed on 12 April 2021). The correlation analysis was performed using the OmicStudio tools, a free online platform for data analysis (https: / www.omicstudio. cn/tool, accessed on 27 September 2021).

\section{Conclusions}

In this study, volatile profiles and flavor properties of PR samples prepared through traditional processing methods were characterized by E-nose, E-tongue, and HS-GC-MS. The total relative content of furans/pyrans increased significantly when the number of cycles increased, while the relative contents of aldehydes and alkanes/alkenes decreased. In addition, alcohols, terpenoids, ketones, sulfides, and nitrogen heterocycles exhibited a tendency of increasing first, and then decreasing and stabilizing. Esters, carboxylic acids, and phenols did not undergo significant changes. The Pearson correlation analysis indicated that the eight volatiles might be significantly correlated with the flavor of the PR samples. The PCA and HCA analysis showed that the number of cycles had a certain effect on the volatile compounds, odor, and taste of PR samples. The RPR sample was significantly different from other PR samples at different processing levels. It is worth noting that additional cycles had little effect on most features when the number of cycles exceeded four.

Therefore, processed PR after four cycles was considered to have similar volatile compounds and flavor characteristics based on the HCA results. The results confirmed the potential applicability of volatile profiles and flavor properties for classification of PR samples with different processing levels. The volatile fingerprint and classification of PR could allow for the selection of samples with specific flavor characteristics based on the food and pharmaceutical applications. Our results provide an effective way to determine the level of PR processing by monitoring the changes of volatile components and flavor characteristics. In conclusion, our study supported the application of this method for discrimination of processed PR samples, and also supported the establishment of similar methods for other traditional Chinese steaming products.

Supplementary Materials: The following are available online. Figure S1: Appearance characteristics of FPR and PR samples with different processing levels, Figure S2: Odor response curves of PR samples with different processing levels, Figure S3: GC-MS profile of PR samples with different processing levels, Figure S4: Mass spectra of 54 identified compounds, Figure S5: Identification of 6 key flavor compounds. (A) Hexanal. (B) 1-Octen-3-OL. (C) Nonanal. (D) Furfural. (E) 2-Furanmethanol. (F) 5-Methylfurfural, Table S1: Sensors used in PEN3 electronic nose and their performance description, Table S2: Odor response values of PR samples with different processing levels, Table S3: Taste response values of PR samples with different processing levels, Table S4: Number of volatile compounds in PR samples with different processing levels, Table S5: Relative content of volatile compounds in PR samples with different processing levels, Table S6: The relative content of common compounds in PR samples with different processing levels, Table S7: The relative content of new compounds in different processing levels, Table S8: The relative content of disappeared compounds in different processing levels, Table S9: List of compounds identified in PR samples with different processing levels.

Author Contributions: Conceptualization, X.C. (Xile Cheng) and X.L.; formal analysis, X.C. (Xile Cheng); investigation, H.J. and S.Q.; resources, X.C. (Xiang Cheng) and S.Q.; data curation, H.J. and T.L.; writing-original draft preparation, X.C. (Xile Cheng); writing-review and editing, D.W. and X.L.; visualization, K.R.; project administration, Y.P.; funding acquisition, X.L. All authors have read and agreed to the published version of the manuscript.

Funding: This work was supported by the National Nature Science Foundation of China (81973465)

Acknowledgments: The authors would like to thank General Manager Zhaoming Li (Liaoning Qingyuan Manchu Medicine Chinese Herbs Planting Co., Ltd.) for providing fresh rhizome samples of Polygonatum sibiricum. 
Conflicts of Interest: The authors declare no conflict of interest.

Sample Availability: Samples of different processing levels are available from the authors.

\section{References}

1. FOC-Flora of China. Polygonatum sibiricum Redouté. Available online: http://www.iplant.cn/foc/ (accessed on 16 July 2021).

2. Pharmacopoeia Commission of the People's Republic of China. Pharmacopoeia of the People's Republic of China (Part I); Chinese Medicine Science and Technology Publishing House: Beijing, China, 2020; pp. 319-320.

3. Zhao, P.; Zhao, C.C.; Li, X.; Gao, Q.Z.; Huang, L.Q.; Xiao, P.G.; Gao, W.Y. The genus Polygonatum: A review of ethnopharmacology, phytochemistry and pharmacology. J. Ethnopharmacol. 2018, 214, 274-291. [CrossRef]

4. Tan, F.; Chen, Y.L.; Tan, X.L.; Ma, Y.Y.; Peng, Y. Chinese materia medica used in medicinal diets. J. Ethnopharmacol. 2017, 206, 40-54. [CrossRef] [PubMed]

5. Yang, Z.Y.; Yang, K.; Zhu, X.X.; Liu, X.M.; Hu, T.; Chen, Y. Development Status and Industry Development Analysis of Polygonati Rhizoma Health-Care Food. J. Hunan Univ. Chin. Med. 2020, 40, 853-859. [CrossRef]

6. Qu, S.H.; Cheng, X.L.; Pan, Y.N.; Ji, H.Y.; Lu, Y.; Li, Z.M.; Liu, X.Q. The history of origin processing and processing methods of Polygonati Rhizoma. J. Shenyang Pharm. Univ. 2020, 37, 379-384.

7. Zhou, L.; Xu, J.D.; Zhou, S.S.; Mao, Q.; Kong, M.; Shen, K.; Li, X.Y.; Duan, S.M.; Xu, J.; Li, S.L. Integrating targeted glycomics and untargeted metabolomics to investigate the processing chemistry of herbal medicines, a case study on Rehmanniae Radix. J. Chromatogr. A 2016, 1472, 74-87. [CrossRef] [PubMed]

8. Li, L.J.; Hong, P.; Chen, F.; Sun, H.; Yang, Y.F.; Yu, X.; Huang, G.L.; Wu, L.M.; Ni, H. Characterization of the Aldehydes and Their Transformations Induced by UV Irradiation and Air Exposure of White Guanxi Honey Pummelo (Citrus grandis (L.) Osbeck) Essential Oil. J. Agric. Food Chem. 2016, 64, 5000-5010. [CrossRef]

9. Wang, Q.; Mu, R.F.; Liu, X.; Zhou, H.M.; Xu, Y.H.; Qin, W.Y.; Yang, C.R.; Wang, L.B.; Li, H.Z.; Xiong, W.Y. Steaming Changes the Composition of Saponins of Panax notoginseng (Burk.) F.H. Chen That Function in Treatment of Hyperlipidemia and Obesity. J. Agric. Food Chem. 2020, 68, 4865-4875. [CrossRef]

10. Cheng, L.Z.; Wang, Y.F.; Zhang, J.R.; Zhu, J.X.; Liu, P.H.; Xu, L.R.; Wei, K.; Zhou, H.; Peng, L.L.; Zhang, J.; et al. Dynamic changes of metabolic profile and taste quality during the long-term aging of Qingzhuan Tea: The impact of storage age. Food Chem. 2021, 359, 129953. [CrossRef] [PubMed]

11. Adelina, N.M.; Wang, H.; Zhang, L.G.; Zhao, Y.H. Comparative analysis of volatile profiles in two grafted pine nuts by headspace-SPME/GC-MS and electronic nose as responses to different roasting conditions. Food Res. Int. 2021, 140, 110026. [CrossRef] [PubMed]

12. Cai, W.C.; Tang, F.X.; Wang, Y.R.; Zhang, Z.D.; Xue, Y.A.; Zhao, X.X.; Guo, Z.; Shan, C.H. Bacterial diversity and flavor profile of Zha-Chili, a traditional fermented food in China. Food Res. Int. 2021, 141, 110112. [CrossRef]

13. Hou, H.; Liu, C.; Lu, X.S.; Fang, D.L.; Hu, Q.H.; Zhang, Y.Y.; Zhao, L.Y. Characterization of flavor frame in shiitake mushrooms (Lentinula edodes) detected by HS-GC-IMS coupled with electronic tongue and sensory analysis: Influence of drying techniques. LWT 2021, 146, 111402. [CrossRef]

14. Kim, M.H.; Lee, S.M.; An, K.W.; Lee, M.J.; Park, D.H. Usage of Natural Volatile Organic Compounds as Biological Modulators of Disease. Int. J. Mol. Sci. 2021, 22, 9421. [CrossRef] [PubMed]

15. Kim, T.; Song, B.; Cho, K.S.; Lee, I.S. Therapeutic potential of volatile terpenes and terpenoids from forests for inflammatory diseases. Int. J. Mol. Sci. 2020, 21, 2187. [CrossRef]

16. Yu, L.; Peng, F.; Xie, J.; Peng, C. Pharmacological Properties of Geraniol-A Review. Planta Med. 2019, 85, 48-55. [CrossRef]

17. Mączka, W.; Wińska, K.; Grabarczyk, M. One hundred faces of geraniol. Molecules 2020, 25, 3303. [CrossRef]

18. Kumawat, V.S.; Kaur, G. Insulinotropic and antidiabetic effects of $\beta$-caryophyllene with l-arginine in type 2 diabetic rats. J. Food Biochem. 2020, 44, e13156. [CrossRef]

19. Qin, Y.; Weng, J.Y.; Pang, Y.M.; Cheng, Z.H. Comparative Analysis of Volatile Organic Compounds in Viola yedoensis by Needle Trap, Static Headspace and Hydrodistillation Methods Coupled with GC-MS. Chin. J. Exp. Tradit. Med. Form. 2019, 25, 153-161. [CrossRef]

20. Kung, T.L.; Chen, Y.J.; Chao, L.K.; Wu, C.S.; Lin, L.Y.; Chen, H.C. Analysis of Volatile Constituents in Platostoma palustre (Blume) Using Headspace Solid-Phase Microextraction and Simultaneous Distillation-Extraction. Foods 2019, 8, 415. [CrossRef]

21. Feng, X.; Shi, H.; Yang, G.Y.; Chang, Y.Q.; Zhang, D.; Zheng, Y.G.; Guo, L. Dynamic changes of volatile components in Forsythia suspensa at different harvest periods based on GC-MS and chemometrics analysis. Chin. J. Chin. Mater. Med. 2020, 45, 8. [CrossRef]

22. Gao, J.C.; Zhou, C.T.; Cheng, Z.H.; Zhang, J.; Tang, A.J. Comparison of Sampling Methods Headspace and SPME GC-MS for Analysis ofVolatile Components in Garlic. Food Res. Dev. 2014, 35, 4. [CrossRef]

23. Lei, L.; Ke, C.; Xiao, K.Y.; Qu, L.H.; Lin, X.; Zhan, X.; Tu, J.Y.; Xu, K.; Liu, Y.J. Identification of different bran-fried Atractylodis Rhizoma and prediction of atractylodin content based on multivariate data mining combined with intelligent color recognition and near-infrared spectroscopy. Spectrochim. Acta A 2021, 262, 120119. [CrossRef] 
24. Ren, X.Y.; He, T.; Wang, J.J.; Wang, L.; Wang, Y.; Liu, X.Y.; Dong, Y.; Ma, J.M.; Jia, J.; Song, R.L.; et al. UV spectroscopy and HPLC combined with chemometrics for rapiddiscrimination and quantification of Curcumae Rhizoma from three botanical origins. J. Pharmaceut. Biomed. 2021, 202, 114145. [CrossRef] [PubMed]

25. Wang, Y.; Zuo, Z.I.; Wang, Y.H. Pattern recognition: An effective tool for quality assessment of herbal medicine based on chemical information. J. Chemom. 2020, 35, e3305. [CrossRef]

26. Cui, S.Q.; Wang, J.; Yang, L.C.; Wu, J.F.; Wang, X.L. Qualitative and quantitative analysis on aroma characteristics of ginseng at different ages using E-nose and GC-MS combined with chemometrics. J. Pharm. Biomed. Anal. 2015, 102, 64-77. [CrossRef]

27. Li, Q.; Yu, X.Z.; Xu, L.R.; Gao, J.M. Novel Method for the Producing Area Identification of Zhongning Goji Berries by Electronic Nose. Food Chem. 2016, 221, 1113. [CrossRef]

28. Wang, Y.; He, T.; Wang, J.J.; Wang, L.; Ren, X.Y.; He, S.H.; Liu, X.Y.; Dong, Y.; Ma, J.M.; Song, R.L. High Performance Liquid Chromatography Fingerprint and Headspace Gas Chromatography-Mass Spectrometry combined with chemometrics for the species authentication of Curcumae Rhizoma. J. Pharm. Biomed. Anal. 2021, 202, 114144. [CrossRef] [PubMed]

29. Guo, M.; Wu, Z.C.; An, Q.; Li, H.Y.; Wang, L.; Zheng, Y.G.; Guo, L.; Zhang, D. Comparison of Volatile Oils and Primary Metabolites of Raw and Honey-Processed Ephedrae Herba by GC-MS and Chemometrics. J. AOAC Int. 2021, 139. [CrossRef]

30. Habinshuti, I.; Mu, T.H.; Zhang, M. Structural, antioxidant, aroma, and sensory characteristics of Maillard reaction products from sweet potato protein hydrolysates as influenced by different ultrasound-assisted enzymatic treatments. Food Chem. 2021, 361, 130090. [CrossRef]

31. Fu, Z.H.; Li, S.J.; Hu, H.H.; Liu, P.; Sun, M.L. Taste comparison of unprocessed and processed Siegesbeckiae puescens based on electronic tongue. Chin. Tradit. Herb. Drugs. 2017, 48, 673-680. [CrossRef]

32. Bai, J.; Gao, L.L.; Zhang, Z.Q.; Cai, M.R.; You, L.T.; Yao, Y.; Wu, H.M.; Yin, X.B.; Ni, J. Application, and related mechanism of electronic tongue technology in traditional Chinese medicine. Cent. South Pharm. 2021, 19, 78-84. [CrossRef]

33. Chai, C.C.; Cao, Y.; Mao, M.; Wang, J.Y.; Liu, N.; Li, X.X.; Zhang, K.; Chen, D.L.; Wei, L.Y.; Yin, Y.H.; et al. Evaluation of taste changes of Scutellariae Radix before and after wine-frying based on electronic ongue technology and its application in identification of Scutellariae Radix pieces. Chin. J. Chin. Mater. Med. 2020, 45, 2552-2559. [CrossRef]

34. Wang, J.; Yue, Y.D.; Tang, F.; Tao, W.Q. Comparative analysis of volatile fractions in Polygonati Rhizoma and its processed products by GC-MS. Chin. J. Chin. Mater. Med. 2012, 36, 18-21.

35. Wei, Z.; Zeng, L.Y.; Song, Z.Q.; Cao, Y.N.; Zhang, L.L.; Liu, Z.L. Comparative Analysis of Chemical Constituent from Polygonati Rhizoma before and After Processing by Headspace Injection-GC-MS. Chin. J. Exp. Tradit. Med. Form. 2012, 18, 115-118. [CrossRef]

36. Wu, Y.; Wang, D.; Guo, L.; Su, J.K.; Ding, Y.P. Changes of Furan Constituents in Rhizome of Three Species of Polygonatum Before and After Processing. J. Chin. Med. Mater. 2015, 38, 1174-1176. [CrossRef]

37. Xu, L.R.; Yu, X.Z.; Li, M.J.; Chen, J.; Wang, X.G. Monitoring oxidative stability and changes in key volatile compounds in edible oils during ambient storage through HS-SPME/GC-MS. Int. J. Food Prop. 2018, 20, S2926-S2938. [CrossRef]

38. Yu, H.Y.; Xie, T.; Xie, J.R.; Chen, C.; Ai, L.Z.; Tian, H.X. Aroma perceptual interactions of benzaldehyde, furfural, and vanillin and their effects on the descriptor intensities of Huangiiu. Food Res. Int. 2020, 129, 108808. [CrossRef] [PubMed]

39. Vargas, R.M.; Munekata, P.; Gagaoua, M.; Franco, D.; Campagnol, P.; Pateiro, M.; Barretto, A.; Domínguez, R.; Lorenzo, J. Inclusion of healthy oils for improving the nutritional characteristics of dry-fermented deer sausage. Foods 2020, 9, 1487. [CrossRef] [PubMed]

40. Ramírez, R.; Cava, R. Volatile profiles of dry-cured meat products from three different Iberian X Duroc genotypes. J. Agric. Food Chem. 2007, 55, 1923-1931. [CrossRef]

41. Amanpour, A.; Guclu, G.; Kelebek, H.; Selli, S. Characterization of key aroma compounds in fresh and roasted terebinth fruits using aroma extract dilution analysis and GC-MS-Olfactometry. Microchem. J. 2019, 145, 96-104. [CrossRef]

42. Papageorgiou, M.; Paraskevopoulou, A.; Pantazi, F.; Skendi, A. Cake perception, texture and aroma profile as affected by wheat flour and cocoa replacement with carob flour. Foods 2020, 9, 1586. [CrossRef]

43. Cho, Y.; Song, M.K.; Kim, T.S.; Ryu, J.C. Identification of novel cytokine biomarkers of hexanal exposure associated with pulmonary toxicity. Environ. Pollut. 2017, 229, 810-817. [CrossRef] [PubMed]

44. Adamová, T.; Hradecký, J.; Pánek, M. Volatile organic compounds (VOCs) from wood and wood-based panels: Methods for evaluation, potential health risks, and mitigation. Polymers 2020, 12, 2289. [CrossRef] [PubMed]

45. Muthumalage, T.; Friedman, M.R.; McGraw, M.D.; Ginsberg, G.; Friedman, A.E.; Rahman, I. Chemical Constituents Involved in E-Cigarette or Vaping Product Use-Associated Lung Injury (EVALI). Toxics 2020, 25, 25. [CrossRef] [PubMed]

46. Liu, D.Y.; Zhou, G.H.; Xu, X.L. ROAV Method: A New Method for Determining Key Odor Compounds of Rugao Ham. Food Sci. 2008, 29, 370-374. [CrossRef]

47. Akkad, R.; Buchko, A.; Johnston, S.P.; Han, J.; House, J.D.; Curtis, J.M. Sprouting improves the flavour quality of faba bean flours. Food Chem. 2021, 364, 130355. [CrossRef] [PubMed]

48. Utrilla, V.M.; Rodríguez, C.J.; Avendaño, C.H.; Gschaedler, A.; Lugo, C.E. Analysis of volatile compounds of five varieties of Maya cocoa during fermentation and drying processes by Venn diagram and PCA. Food Res. Int. 2020, 129, 108834. [CrossRef] 